\title{
Coronavirus (COVID-19): A Patient Experience - Administrative services on the frontline during crisis
}

\author{
Randal S. Weber, MD ${ }^{1}$ \\ ${ }^{1}$ The University of Texas MD Anderson Cancer Center
}

April 29, 2020

\section{Judy Overton, BFA, MLA}

Program Manager, Office of Patient Experience Officer

The University of Texas M.D. Anderson Cancer Center

T. Boone Pickens Academic Tower

1400 Pressler St

Houston, Texas 77030

\section{Kathy Denton, BS, MED, PhD}

The University of Texas M.D. Anderson Cancer Center, Director, Patient Experience

The University of Texas M.D. Anderson Cancer Center

T. Boone Pickens Academic Tower

1400 Pressler St

Houston, Texas 77030

Michael Frumovitz, MD, MPH

Gynecologic Oncology and Reconstructive Medicine

The University of Texas M.D. Anderson Cancer Center

Dan L. Duncan Building

1155 Pressler St

Houston, Texas 77030

Carol Lewis, MD 
Department of Head and Neck Surgery

The University of Texas M.D. Anderson Cancer Center

T. Boone Pickens Academic Tower

1400 Pressler St

Houston, Texas 77030

\section{Sarah Christensen, MA}

Director, Patient Education

The University of Texas M.D. Anderson Cancer Center

Dan L. Duncan Building

1155 Pressler St

Houston, Texas 77030

\section{Jaymesson Bezerra, MSHA}

Manager, Patient Relations

The University of Texas M.D. Anderson Cancer Center

Rotary House International

1600 Holcombe Blvd

Houston, Texas 77030

\section{Chris Hernandez}

Exec. Director, Patient Services, Patient Advocacy

The University of Texas M.D. Anderson Cancer Center

Anderson West

1515 Holcombe Blvd

Houston, Texas 77030

\section{Michele S. Walker}

Assoc. Dir, Pt Advo \& Pt Rel, Patient Advocacy

The University of Texas M.D. Anderson Cancer Center

Anderson West

1515 Holcombe Blvd

Houston, Texas 77030 
Janice P. Finder, BSN, MSN, RN

Dirrector, Patient Exp Clin Services, askMDAnderson

The University of Texas M.D. Anderson Cancer Center

Mid Campus Building 1MC

7007 Bertner Avenue

Houston, Texas 77054

\section{Ashlyn A. Proske, BS}

Program Manager, askMDAnderson

The University of Texas M.D. Anderson Cancer Center

Mid Campus Building 1MC

7007 Bertner Avenue

Houston, Texas 77054

\section{Sanchita Jain, MBA}

Innovation Strategist, Innovation

The University of Texas M.D. Anderson Cancer Center

Mid Campus Building 1MC

7007 Bertner Avenue

Houston, Texas 77054

\section{Julai Whipple, BA}

Innovation Designer, Innovation

The University of Texas M.D. Anderson Cancer Center

Mid Campus Building 1MC

7007 Bertner Avenue

Houston, Texas 77054

\section{Wendi L. Martinez, ADN, BSN, RN}

Dir QA \& Perf Improvement, Inst Cancer Care Innovation The University of Texas M.D. Anderson Cancer Center

Mid Campus Building 1MC

7007 Bertner Avenue

Houston, Texas 77054 


\section{Jarod Eska}

Inst Cancer Care Innovation

The University of Texas M.D. Anderson Cancer Center

Mid Campus Building 1MC

7007 Bertner Avenue

Houston, Texas 77054

\section{Elizabeth W. Sutherland, MPAS}

Physician Asst, Surgical Oncology

The University of Texas M.D. Anderson Cancer Center

T. Boone Pickens Academic Tower

1400 Pressler St.

Houston, Texas 77030

\section{Lisa L. Triche, DNP, MS, RN}

Advanced Prac Registered Nurse, Pediatrics - Patient Care

The University of Texas M.D. Anderson Cancer Center

Anderson East

1515 Holcombe Blvd

Houston, Texas 77054

\section{Elizabeth A. Garcia, BSN, MPA, RN}

Assoc VP, Patient Experience, Ofc of Chief Operating Officer

The University of Texas M.D. Anderson Cancer Center

1515 Holcombe Blvd

Unit 1485

Houston, Texas 77030

\section{Corresponding Author:}

\section{Randal Weber, MD}

Chief Patient Experience Ofc, Chief Patient Experience Officer

The University of Texas M.D. Anderson Cancer Center

T. Boone Pickens Academic Tower

1400 Pressler St

Houston, Texas 77030 


\section{Introduction}

The COVID-19 pandemic has had a dramatic impact on care delivery among health care institutions and providers in the United States. Prioritization of scarce health care resources for those afflicted by the COVID-19 infection has disrupted delivery of health care mandating a reallocation of care to patients with urgent needs. Important goals of care are timeliness, effective treatments, and ready access. While these are critical goals, they have been redirected towards acutely ill patients in urgent need of care. As a categorical cancer center, we have had to prioritize care for our patients based on acuity of their disease, protect patients and employees from acquiring the infection within the confines of our facilities, and provide acute management of cancer patients with concomitant COVID-19 infections who are considered at high risk of death.

\section{Background}

In October 2016, MD Anderson formally established a division of Patient Experience under the dyad leadership of a physician (RSW) and a nurse administrator (EG). Working with their dedicated team, the patient experience program developed key strategic initiatives to create a culture of patient-centered care. Several process improvement measures were implemented using CAHPS data to identify opportunities to create a new culture of patient centeredness. In 2018, Peter Pisters, M.D., the President of MD Anderson, transitioned the patient experience program from an operational priority to a cornerstone of administrative and operational priorities for allocation of resources to support cultural change.

What follows is a summary of the patient experience team's effort to actively support programs and processes in anticipation of the pandemic's effect on our patients and employees. We will describe how the team continues to serve in the ever-dynamic environment as we approach the expected surge in COVID-19 cases among our patient population, our employees, and in our community.

\section{Preparing for the COVID-19 threat to MD Anderson}

COVID-19 first appeared in November of 2019 and quickly overwhelmed China's health care system. ${ }^{1}$ Subsequently, the virus spread around the globe creating casualties that numbered in the thousands in a matter of weeks. The reality of COVID-19's threat to the United States occurred in late January 2020 with the report of the first death on the eastern seaboard. The virus has spread across the country with various municipalities experiencing sequential surges in their afflicted population.

Anticipating a rising incidence of infections in the Houston metropolitan region, MD Anderson's leadership took immediate steps to proactively protect our patients who are highly susceptible to infection and severe complications secondary to their compromised immunity.

Patient and employee screening were rapidly implemented to identify those at risk for exposure to COVID19 or actively infected by it. Screening criteria included travel history, exposure to infected individuals, fever, cough or breathing difficulties, as well as temperature checks. Immediate travel restrictions were implemented for all employees.

\section{Implementing a New Normal}

The Chief Quality Officer (CQO) quickly recognized the need to manage the traffic into the institution as a strategy to prevent spread of infection among our vulnerable patients. Early on, the CQO led a site visit throughout the institution to determine which entrances would be used to screen patients, visitors, and employees. The institution limited points of access for employees and segregated patient/caregiver and employee access points to facilitate screenings.

The Patient Relations Team initially provided scheduling support and oversight of the access point screeners. Twenty-one employees staffed the frontline entryways for 12-hour shifts, Monday through Friday. Leadership quickly realized this was not sustainable. In order to avoid screener fatigue, they quickly trained additional screeners from the institutional workforce to support the screening needs at access points. 
When data collection began on March 17, 2020, the screeners saw an average of 8,944 employees and 2,793 patients/visitors per day. After initiating remote work options (implemented to insure only essential employees were on site) and a zero visitor policy with minimal exceptions on March 24, the screeners saw an average of 4,849 employees and 1,049 patients/visitors per day.

With the increase in reassignments of essential employees only onsite, the available screeners decreased significantly. To provide adequate screening personnel, the Department of Human Resources (HR) assumed responsibility for providing the work force at the points or access. The transition to HR allowed continued employment for staff, such as those in research or administrative support, who might have required furlough due to decreased clinical activity from COVID-19 preparedness.

\section{Enhanced vigilance, compassionate care}

Throughout the month of March, the COVID-19 alerts quickly escalated. To protect our vulnerable patients and employees in an attempt to limit exposure in the face of community spread, executive leadership issued a series of rapid changes to our visitor policy. On March 9, the policy was adjusted to allow only two visitors to enter an inpatient room or accompany a patient to an ambulatory center. Two days later, the policy was adjusted to permit only adult visitors. On March 19, the institution changed this to a one visitor policy, and on March 24, this was further limited to a no visitor policy. Exceptions were granted on a case-by-case basis for patients with cognitive and self-care issues and those facing end of life.

The institution continually informed patients about the dynamic policy changes via the electronic health record patient portal and on the public-facing MD Anderson website. Still, many patients arrived with their caregivers, hoping to be granted an exception to the policy.

The onsite Patient Advocate, along with frontline staff, provided the important rationale for these restrictions, and if appropriate, sought assessment of exceptions from the nursing and medical teams. The message was delivered with compassion and professionalism to our patients and their families, explaining that we were placing their safety first and foremost.

Exemptions to the no visitor policy were granted based on the detailed criteria developed by the executive leadership team. The nursing teams, in close collaboration with the attending physician, were charged with assessing if a patient met the exception criteria. This shared decision-making empowered those providing care on the front line to mitigate the risk of exposure for patients and staff to visitors who may have an asymptomatic infection with the virus.

At times, patients and family members disagreed with the exception criteria set by the institution. In these cases, patient advocates facilitated the Chief Quality Officer's review of the case. Virtual family care conferences were held with involved caregivers to provide a thorough explanation of the criteria and to work through communication accommodations, such as video conferencing with family members and care teams.

The institution developed a process to ease the impact of the no visitor policy on patients and their loved ones. Nursing leaders were available at each entrance to assist, in real-time, with any difficult conversations, and reassigned employees were available to escort a number of patients to their appointments.

\section{Clear, concise and thorough communication}

Clinical teams in the askMDAnderson call center were made available 24/7 to inform patients about COVID19 testing sites. They scheduled patient appointments to testing sites, reviewed their test results, and ensured patients received urgent medical care in the right place and at the right time.

Consistent messaging to internal and external stakeholders was maintained through a collaborative effort between Patient Education and Strategic Communications. Together, the teams developed consistent dynamic content for both patients and employees. The process typically originated in brainstorming sessions with clinical working groups in which processes were broken down into the elements of utmost importance to patients and their caregivers. 
As a result, Patient Education has produced numerous COVID-19-related information materials that are available to all stakeholders:

- Coronavirus Disease (COVID-19) Screening Next Steps

- Self-Quarantine Instructions (in English and Spanish)

· MyChart Video Visit Instructions (English and Spanish)

· $\quad$ MyChart Video Visit Instructions (online video)

Additional documents and videos remain in production to address the dynamic changes and offerings in processes and are being disseminated upon completion.

To facilitate the need to frequently update content, Patient Education materials were made available via URL rather than using PDFs. In addition, Strategic Communications established a website dedicated to the dissemination of MD Anderson's COVID-19 response.

\section{Technology-based creative solutions}

MD Anderson rapidly implemented a combination of virtual care solutions for our patients, families, and providers to meet a range of needs in situations when in-person visits are not possible or ill-advised. The institution had already moved to implement virtual care, but the onset of COVID-19 accelerated the needs to use these various options.

While some clinicians continue to see patients in outpatient clinics and inpatient settings, the institution launched the use of MyChart video visits, an online telemedicine tool that combines Epic (Verona, WI) and Zoom (San Jose, CA) to decrease the number of people who entered our facilities. This option protected our patients who are at very high risk because of their cancer, as well as our employees. ${ }^{2}$ Providers and conducted more than 2,000 scheduled visits within the first three weeks of the launch of the MyChart virtual visits.

The Virtual Care Task Force, a multidisciplinary team led by MD Anderson's Innovation team, studied the use of the technology and launched a virtual care solution, with the approval of executive leadership. The virtual care program is meant for follow-up appointments, consults, and research study coordination across several specialties. It also meets Center for Medicare Services criteria for billing and offers inbuilt billing technical support in an environment of constantly shifting legal requirements. The suite of solutions offers provisions for patients to connect with their socially distanced families and video monitoring technology for admitted patients under investigation (PUI) for a potential COVID-19 infection.

All disease sites were included in the launch of the virtual visits. Standardized patient satisfaction surveys are being implemented to evaluate, obtain feedback, and continually improve the value of video visits.

The Virtual Care Task Force is also responsible for the implementation of Vivify Health, a remote patient monitoring application utilized to virtually monitor COVID-19 symptoms. The app serves as a guide for new out-of-state patients with future appointments at MD Anderson who are required to self-quarantine, with monitoring and reporting of symptoms, for 14 days before their initial visits. The quarantine is waived for the patient to receive robust symptom assessment and COVID-19 testing when the medical condition warrants it. Patients monitor their health with the guidance of a case manager who will conduct a virtual check-in with the patient twice per day, at 9 a.m. and 5 p.m. This is a free, optional service and can be helpful for those patients who may not have access to virtual care services through their primary care physicians or those who do not have a primary care physician. The institution is looking to future use of the remote patient monitoring application for discharged patients.

\section{Triaging surgeries and access to care}

Overarching goals for managing patient access to the institution included delivering care to those whose disease required rapid intervention, while conserving resources (PPE, hospital beds, etc.), in anticipation of 
a surge in COVID-19 infected patients who might require inpatient care. The criteria for current onsite appointments included new and existing local patients (those within 150 miles of the Houston) and those with rare cancer tumors, without geographic restriction.

In accordance with state mandates, elective surgeries, including those for benign disease, have been postponed to make hospital beds available in anticipation of a surge in COVID-19 diagnoses among our cancer patients. Urgent or emergent surgeries still occur after a careful daily review of prospective procedures based on specific prioritization criteria. A multidisciplinary review committee carefully evaluates each posted procedure, factoring in disease-specific factors, historical data on transfusion requirements, the likelihood of Intensive Care Unit (ICU) admission based on procedure type, as well as the potential need for other resources, including personal protective equipment.

\section{Adjusting State Mandates for our Patients}

As of March 22, 2020, Texas Governor Greg Abbott issued a mandate that, effective March 23, all travelers from national hotspots, including our neighboring state of Louisiana, had to self-quarantine for 14 days upon arrival to Texas. At this time, many existing MD Anderson patients were traveling from these locations for planned appointments and surgeries. The quarantine not only imposed delays in care, but required patients to find housing and resources in Texas for 14 days before receiving care. Within days, MD Anderson created a policy that patients can self-quarantine at home or a hotel before traveling to MD Anderson for appointments. A team was created to follow and check on these patients before their travel to and upon arrival to MD Anderson.

\section{Advancing the use of the Advance Care Plan}

The pandemic has elevated the patient and family experience for those who are at the end of life by centering on the patient's goals of care (GOC) and presenting an opportunity for the institution to be very clear on available treatments. These measures were implemented to provide clarity on the GOC and to prevent unnecessary admissions to the inpatient setting or escalation to the intensive care unit when not consistent with the disease state and patient's agreed upon GOC.

Supportive Care leadership heads a Goals of Caring (GOC) Rapid Response Team (RRT) that has developed a strategy in response to the crisis and is comprised of three components: 1 . deploy, at short notice, a Supportive Care faculty to join an oncologist in the outpatient or inpatients setting to help conduct GOC conversation; following a short briefing, both physicians enter the room and conduct the GOC talk, following a specially-developed check list; 2 . implemented a GOC rapid educational program with formal live education for oncologists, five different scripts to cover multiple inpatient and outpatient scenarios, a daily drop-in, onehour coaching clinic, and five minute mini-tutorials; and 3. provide a daily review of all inpatient admissions and all ICU admissions to identify patients who may quality for the GOC RRT and to educate the primary oncologist on the importance of anticipating these events with GOC conversations.

\section{Conclusions}

MD Anderson institutional leadership set forth priorities to protect patients and staff while minimizing disease impact on our community. As patients and staff adjust to sweeping changes that serve these goals, our patient experience team has proven both instrumental in providing clear and effective communication with patients and critical in advocating for patients and addressing their concerns and grievances. Our patient advocates were quick to offer options to family members to engage with their loved ones through video, FaceTime and updates from the nurse or a chaplain. We gained insights on making adjustments that will benefit all of our stakeholders in this challenging situation.

Throughout this process, employees, particularly those who are consistently on the frontline in direct and indirect encounters with patients and caregivers, have demonstrated resoluteness, caring, and compassion. In 
these unprecedented and uncertain times, each member of the MD Anderson family, our patients and their loved ones, have all proven to exhibit extraordinary resilience.

\section{References}

$1^{\text {st }}$ known case of coronavirus traced back to November in China. LIVESCIENCE. March 14, 2020. https: //www.livescience.com/first-case-coronavirus-found.html, Accessed April 23, 2020.

COVID-19 hits doctors, nurses, EMTs. The Washington Post, March 17, 2020. Accessed Saturday, April 11, 2020 . 